University of Nebraska - Lincoln

DigitalCommons@University of Nebraska - Lincoln

June 2006

\title{
Biochemical basis of specialization for dispersal vs. reproduction in a wing-polymorphic cricket: Morph-specific metabolism of amino acids
}

Zhangwu Zhao

University of Georgia, zhaozw@uga.edu

Anthony J. Zera

University of Nebraska - Lincoln, azera1@unl.edu

Follow this and additional works at: https://digitalcommons.unl.edu/bioscizera

Part of the Microbiology Commons

Zhao, Zhangwu and Zera, Anthony J., "Biochemical basis of specialization for dispersal vs. reproduction in a wing-polymorphic cricket: Morph-specific metabolism of amino acids" (2006). Anthony Zera

Publications. 3.

https://digitalcommons.unl.edu/bioscizera/3

This Article is brought to you for free and open access by the Papers in the Biological Sciences at DigitalCommons@University of Nebraska - Lincoln. It has been accepted for inclusion in Anthony Zera Publications by an authorized administrator of DigitalCommons@University of Nebraska - Lincoln. 


\title{
Biochemical basis of specialization for dispersal vs. reproduction in a wing-polymorphic cricket: Morph-specific metabolism of amino acids
}

\author{
Zhangwu Zhao* and Anthony J. Zera ${ }^{\dagger}$ \\ School of Biological Sciences, University of Nebraska-Lincoln, Lincoln, NE 68588 \\ $\dagger$ Corresponding author. E-mail: azera1@unlnotes.unl.edu \\ *Current address: Department of Cellular Biology, University of Georgia, 724 Biological \\ Sciences Building, Athens, GA 30602. Email: zhaozw@uga.edu
}

\begin{abstract}
The biochemical basis of specializations for dispersal vs. reproduction is an understudied aspect of dispersal polymorphism in insects. Using a radiolabelled amino acid, we quantified differences in in vivo amino acid metabolism between morphs of the wing-polymorphic cricket, Gryllus firmus, that trade-off early age reproduction and dispersal capability. Studies were conducted in crickets fed a variety of diets expected to influence amino acid and lipid metabolism. On the day of molt to adulthood, prior to the morph-specific trade-off between ovarian growth and biochemical preparation for flight (e.g. biosynthesis of triglyceride flight fuel), morphs did not differ in any aspect of amino acid metabolism. However, on day 5 of adulthood, when the morph-specific trade-off between ovarian growth and flight fuel production was manifest, the morphs differed substantially in each of the three aspects of amino acid metabolism studied: conversion to protein, oxidation, and conversion to lipid. Morphs also differed in degree of allocation of products of amino acid metabolism to ovaries vs. the soma. Most importantly, morphs differed in the relative metabolism of radiolabelled glycine through these pathways (i.e. biochemical trade-offs), and in the relative allocation of end products of amino acid metabolism to the soma vs. ovaries (allocation trade-offs). A functionally important interaction between amino acid and lipid metabolism was noted: greater oxidation of amino acids in the flight-capable morph spared fatty acids for enhanced conversion into triglyceride flight fuel. By contrast, greater oxidation of fatty acids by the flightless morph spared amino acids for enhanced conversion into ovarian protein. Diet significantly affected amino acid metabolism. However, MORPH $\times$ DIET interactions were rare and morphs differed in amino acid metabolism to a similar degree under the range of diets tested.
\end{abstract}

Keywords: Trade-off, Wing polymorphism, Life history, Dispersal, Intermediary metabolism, Amino acid

\section{Introduction}

Wing (dispersal) polymorphism occurs widely in the Insecta and involves discontinuous variation in a wide variety of traits (e.g. wing length, flight muscle mass, ovarian mass, flight fuel concentration) resulting in morphs that are specialized for flight at the expense of reproduction and vice versa (Harrison, 1980; Socha, 1993; Dingle, 1996; Zera and Denno, 1997; Zera and Harshman, 2001). The polymorphism has been used extensively as a model to investigate a wide variety of issues relating to the evolution, ecology, and physiology of dispersal, reproduction, and their negative interaction (trade-off) (Harrison, 1980; Roff, 1986 and Roff, 2002; Dingle, 1996; Zera and Den- no, 1997; Tanaka, 1994; Socha and Kodrik, 1999; Zera and Harshman, 2001; Zera, 2004, Zera, 2005 and Zera, 2006). A number of physiological studies have documented differences between morphs in energetic or endocrine aspects of flight capability and egg production (reviewed in Zera and Denno, 1997; Zera et al., 1998; Zera and Harshman, 2001, Zera, 2004, Zera, 2005 and Zera, 2006). More recently studies in the wing-polymorphic cricket, Gryllus firmus, have begun to investigate the biochemical basis of morph specialization for dispersal vs. reproduction, most notably the role of morph-specific differences in lipid metabolism (Zhao and Zera, 2002; Zera and Zhao, 2003a, Zera and Zhao, 2003b, Zera and Zhao, 2004 and Zera and Zhao, 2006; Zera, 2005 and Zera, 2006). 
As is the case for many other insects, especially orthopterans (Beenakkers et al., 1985; Candy, 1989), the flight-capable morph of G. firmus accumulates substantially more total lipid and triglyceride, than its flight-capable counterpart during the first week of adulthood (Zera and Larsen, 2001). Subsequent biochemical studies have shown that the increased lipid (triglyceride) levels in the dispersing morph are produced by elevated de novo biosynthesis of fatty acids, increased diversion of fatty acids into triglyceride as opposed to phospholipid biosynthesis, and decreased oxidation of fatty acids relative to the flightless morph (Zhao and Zera, 2002; Zera and Zhao, 2003a and Zera and Zhao, 2003b; Zera, 2005).

Alterations in amino acid metabolism are also expected to play an important role in morph specialization for flight vs. reproduction. Amino acids can be metabolized by a variety of pathways (Candy, 1985; Clements, 1959; Stryer, 1988), whose products can contribute importantly to flight or reproductive functions (Boggs, 1992; O'Brien et al., 2002 and O'Brien et al., 2005). Amino acids are precursors of somatic (e.g. flight muscle) and reproductive proteins (e.g. vitellogenin) (path-way 1, Fig. 1a); they can also serve as an important energy source via conversion to Krebs Cycle in-

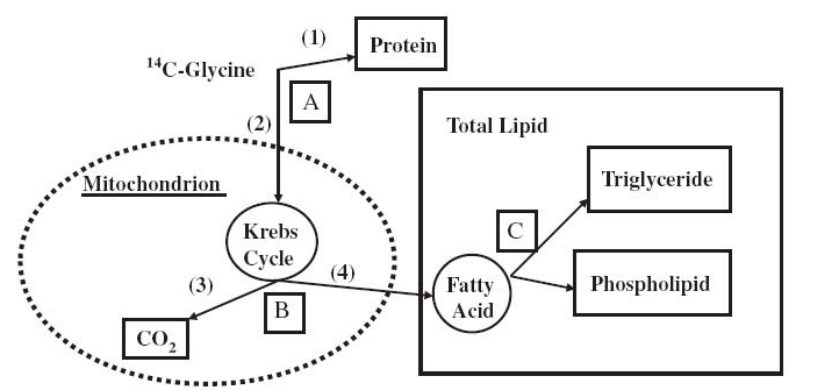

(a)

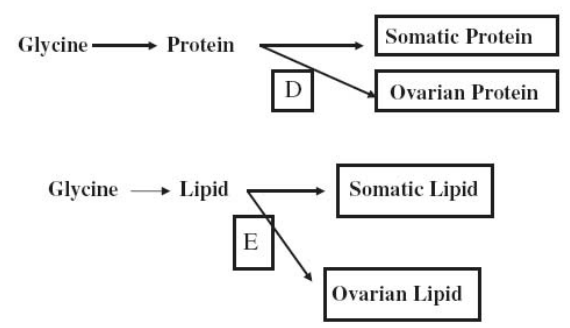

(b)

Figure 1. (a) Metabolic fates of the amino acid glycine, investigated in the present study: (A) utilization for protein biosynthesis (pathway 1), (B) oxidation to $\mathrm{CO}_{2}$ via the Krebs cycle (pathways $2+3$ ), and $(\mathrm{C})$ transformation into lipid (pathways 2+4). Other metabolic fates are possible (e.g. conversion into carbohydrate or diversion through other pathways in the Krebs Cycle), but were not investigated. Boxed metabolites were quantified, while encircled intermediates were not. Letters A-C denote the three metabolic trade-offs investigated. Values (proportional diversion of radiolabel) for these trade-offs are given in Table 3 and Table 4. (b) Allocation trade-offs (proportional diversion of end products of metabolism to ovaries vs. soma (whole body-ovaries)). Values for these trade-offs are given in Table 3 and Table 4. termediates (pathway 2), and subsequent oxidation to $\mathrm{CO}_{2}$ (Clements 1959) (pathway 3, Fig. 1a); finally, amino acids can be transformed, via the Krebs Cycle, into a variety of somatic or reproductive macromolecules such as lipid (Kon and Monroe, 1971) (pathway 4, Fig. 1a). Although morph specializations for dispersal vs. reproduction are expected to require changes in the relative flux through these pathways, no study has reported on this topic for any wing-polymorphic insect. In the present study we used a radiolabelled amino acid to investigate the relative in vivo flux through the three main pathways of amino acid metabolism described above in the flight-capable and flightless morphs of G. firmus. This study is part of a larger investigation of the genetic and environmental influences of the biochemical basis of the dispersal-reproduction trade-off (reviewed in Zera, 2005; Zera and Zhao, 2006). Here we present data on unselected control populations of G. firmus in which morphs were fed a variety of diets expected to influence amino acid and lipid metabolism. Morph-specific amino acid metabolism was measured prior to and during the period when ovarian growth tradesoff with flight fuel production in the morphs. In a companion study, we investigated the genetic basis of morph differences in amino acid metabolism in artificially selected lines raised on a single (standard) diet (Zera and Zhao, 2006).

\section{Materials and methods}

\subsection{Background on G. firmus: morphs, selected lines and rearing conditions}

G. firmus, the sand field cricket, occurs in natural populations in the southeastern United States as a long-winged (LW) morph, some of which are capable of flight, or as a short-winged (SW) morph that is obligatorily flightless (Veazy et al., 1976; Zera et al., 1997). Except in a few rare cases, all SW females molt into adults with white, nonfunctional, underdeveloped flight muscles. All LW females initially have fully developed (pink) flight muscles at or shortly after the adult molt [denoted LW(f)]; however, flight muscles degenerate in some of these females rendering them flightless. The stocks used in the present study contained a low frequency of LW females with histolyzed flight muscles during the period of adulthood (first week) studied $(<10 \%)$, and the LW-flightless morph is not considered here (see Zera et al., 1997; Zhao and Zera, 2001 for physiological and biochemical information on this morph).

Glycine metabolism was measured in females from two unselected, control lines that were part of an artificial selection study. The selection study consisted of three blocks (independent selection trials), each of which contains a line selected for the LW(f) morph and a line selected for the SW morph. Two of the blocks ( 1 and 2 ) had unselected control lines. In these control lines an equal number of LW and SW males and females were bred each generation. This breeding design was 
used rather than randomly choosing individuals as breeders, because SW females produce about $100-400 \%$ more eggs than LW females (see Section 3). Thus, randomly choosing females as breeders would result in the fixation of the SW morph over time. All lines had been derived from one and same base population, which, in turn was initiated from crickets collected in Gainesville, Fla. (see Zera and Cisper (2001), and Zera (2005) for details of artificial selection). All lines had been raised under the same temperature $\left(28 \pm 1^{\circ} \mathrm{C}\right)$, photoperiod $(16$ light: 8 dark), and had been fed the standard (100\%) diet since their inception. Artificially selected lines have been extensively characterized with respect to aspects of lipid metabolism and life history evolution (Zera and Bottsford, 2001; Zera and Cisper, 2001; Zera and Zhao, 2003a and Zera and Zhao, 2006; Zhao and Zera, 2002; Zera, 2005) (see Section 4).

The main focus of the present study was the C- 1 line, in which amino acid metabolism was compared between the LW(f) and SW morphs at two points in adulthood (days 0 and 5) in individuals fed three diets (described below). Morphs of a second control line (C-2) were studied under a more restricted set of conditions (amino acid metabolism measured on day 5 of adulthood in crickets fed the standard diet), mainly to determine the reproducibility of results obtained for the C-1 line for the most important environmental condition. A companion study (Zera and Zhao, 2006) reports genetic differences in amino acid metabolism in the three pairs of artificially selected lines described above measured in day-5 adults fed the standard diet (see Section 4). Only female morphs were measured in these studies, as was the case for previous studies of lipid metabolism (Zera, 2005).

\subsection{Diets}

Starting on the day of molt to adulthood, C-1 crickets were fed one of three diets: (1) the standard high-nutrient (100\%) diet, (2) a low-nutrient $(25 \%)$ diet, in which $75 \%$ of the nutrients of the standard diet had been removed and substituted with nonnutritive cellulose, and (3) a high carbohydrate diet (50\%), in which $50 \%$ of the nutrients of the standard diet had been removed and substituted with sucrose. These diets are known to affect whole-body mass gain, ovarian growth, lipid accumulation, and many aspects of lipid metabolism in morphs of Gryllus (Zera et al., 1998; Zera and Larsen, 2001; Zera and Zhao, 2003a and Zera and Zhao, 2003b). C-1 crickets were raised on the high nutrient $(100 \%)$ diet from egg hatch until molt to adulthood (day 0), after which they were either maintained on that diet, or switched to one of the other two diets; C-2 crickets were always fed the standard $100 \%$ diet.

\subsection{Incorporation of ${ }^{14} \mathrm{C}$-glycine into lipid classes, $\mathrm{CO}_{2}$, and protein}

Methods used to measure morph-specific incorporation of radio-labelled carbon from ${ }^{14} \mathrm{C}$-glycine into lipids, $\mathrm{CO}_{2}$ and protein were identical to those described in Zera and Zhao (2006). Briefly, two $\mu \mathrm{L}$ of sterile saline $(0.9 \% \mathrm{NaCl})$ containing $8.8 \times 10^{5} \mathrm{DPM}$ of $\left[1-{ }^{14} \mathrm{C}\right]$-glycine $(4.2 \mathrm{GBq} / \mathrm{mmol}$; $112.7 \mathrm{mCi} / \mathrm{mmol}$ ) were injected into the abdominal hemocoel of adult female crickets that had been starved for $4 \mathrm{~h}$. Crickets were placed one individual per $50 \mathrm{ml}$ erlenmeyer flask. Standard chemical extraction protocols, thin-layer chromatography, and liquid scintillation counting (see Zera and Zhao (2006) for details) were used to quantify the amount of radiolabel incorporated during a $5 \mathrm{~h}$ incubation period $\left(28^{\circ} \mathrm{C}\right)$ into the following chemical components: expired $\mathrm{CO}_{2}$ (trapped in aqueous $\mathrm{NaOH}$ contained in a cup near the top of the sealed flask); ovarian and somatic (whole-body minus ovaries) total protein; and somatic and ovarian total lipid, triglyceride and phospholipid. Incorporation of ${ }^{14} \mathrm{C}$ from glycine into carbohydrate was not measured because carbohydrate levels are low in morphs of G. firmus and do not appear to contribute significantly to the trade-off between early age reproduction and flight capability (Zera and Larsen, 2001).

\subsection{Experimental strategy and rational}

This study consisted of two main parts, each of which had two components. In the first part we tested whether LW(f) and SW morphs differ in (a) whole-organism flux (rate) through individual pathways of amino acid metabolism, and (b) allocation of end products of metabolism to individual body compartments (amount of metabolic products accumulated in ovaries or soma (body minus ovaries) during the assay period). In the second part, we investigated morph-specific tradeoffs in (a) proportional flux through bifurcating metabolic pathways (metabolic trade-offs), and (b) proportional allocation of metabolic products to the soma vs. ovaries (allocation trade-offs), using data obtained in part one. We quantified both morph-specific differences in flux through individual pathways, as well as differences in the extent to which end products of these pathways are allocated to somatic vs. reproductive body compartments, because both of these factors are key biochemical components of life history adaptations. For example, amino acids can be transformed into triglyceride, which can function as either a somatic (flight fuel; Zera et al., 1999) or as a reproductive energy reserve, depending upon whether biosynthesized triglyceride is transported to somatic or reproductive body compartments.

In the first part of the study, we quantified the amount of radiolabelled carbon from injected ${ }^{14} \mathrm{C}$-glycine that was incorporated into (a) protein (utilization of amino acid for protein biosynthesis; pathway 1, Fig. 1a), (b) $\mathrm{CO}_{2}$ (amino acid oxidation for energy production; pathway $2+3$ ), and (c) lipid (conversion of amino acid into total lipid and various functionally important lipid components; pathway 2+4). We first tested for morph-specific differences in whole-organism rates of production of individual metabolic products (e.g. protein or lipid) from glycine by pooling 
metabolite data from ovaries and soma (statistical analysis described below). We then tested for differences between the morphs in compartment-specific allocation of end products by comparing the morphs with respect to the quantity of various end products found in the ovaries or soma.

Although amino acids can be converted to a variety of important macromolecules (e.g. lipid, carbohydrate), lipid was the focus of conversion of amino acid into a non-protein constituent. This was done because a large proportion of amino acid can be converted into lipid (see Introduction), and lipid is composed of several key constituents (triglyceride and phospholipid), each of which can have important somatic or reproductive functions (e.g. triglyceride is the main flight fuel in Gryllus; Zera et al., 1999). Large magnitude differences in lipid metabolism have previously been documented between morphs of G. firmus (Zhao and Zera, 2002; Zera and Zhao, 2003a and Zera and Zhao, 2003b; Zera, 2005).

The focus of the second part of the study was morph-specific metabolic and allocation trade-offs. Metabolic tradeoffs (denoted by capital letters in Fig. 1a) are defined as morph-differences in the proportional, whole-organism production of one of a pair of metabolites of a bifurcating metabolic pathway. Allocation trade-offs (denoted by capital letters in Fig. 1b), by contrast, are defined as the proportion of a particular end product of metabolism that is found in one of two body compartments (e.g. soma/(soma+ovaries)). For example, we investigated whether morphs differed in the proportion of glycine that was used for protein biosynthesis vs. converted through the Krebs Cycle into non-protein constituents $\left(\mathrm{CO}_{2}\right.$ plus lipid; Trade-off "A" in Fig. 1a), or the proportional allocation of biosynthesized protein to somatic vs. ovarian body compartments (trade-off " $D$ " in Fig. 1b). It is important to note that the extent to which morphs differ in flux through a single pathway or allocation to a single body compartment is independent of whether they differ in proportional metabolism through linked pathways or proportional allocation to different body compartments. Statistical analyses of these trade-offs are discussed below.

Estimating various trade-offs and testing their statistical significance required pooling data on radiolabel incorporation in various ways, which was determined by whether metabolic or allocation trade-offs were being tested, and specific aspects of the pathways being compared (see Fig. 1a). Because metabolic trade-offs were defined in terms of whole-organism production of metabolites, these tradeoffs always involved pooling data from somatic and ovarian body compartments to obtain whole-organism values. By contrast, allocation trade-offs, which were defined as the proportional diversion of a single metabolic product to different body compartments, never involved data pooling. Other types of pooling were required to test specific tradeoff hypotheses. For example, the first metabolic trade-off tested, was the proportional diversion of carbon from glycine into protein, vs. non-protein metabolites (i.e. carbon derived from glycine metabolized through the Krebs Cycle to produce $\mathrm{CO}_{2}$ and lipid; Trade-off "A"; Fig. 1a). To test for the existence of trade-off "A" we compared radiolabelled whole-body (somatic+ovarian) protein vs. non-protein metabolites $\left(\mathrm{CO}_{2}+\right.$ total lipid in ovaries and soma), in LW(f) vs. SW morphs (see Table 3 for calculation of proportions). The next metabolic trade-off ("B" Fig. 1) involves the morph-specific proportional diversion of radiolabel from glycine into $\mathrm{CO}_{2}$ vs. total (somatic plus ovarian) lipid (i.e. the relative oxidation of glycine for energy production vs. conversion into all lipid components). The final metabolic trade-off tested consisted of morph-specific, proportional diversion of radiolabel from glycine into total (whole-body) triglyceride (energy storage) vs. total phospholipid (structural lipid). Two morph-specific, organ-allocation trade-offs, involving the proportional incorporation of protein ("D"), or total lipid ("E"), biosynthesized from glycine, into ovarian vs. somatic body compartments were tested. These allocation trade-offs are illustrated in Fig. 1b, and calculation of proportions is described in Table 3 .

Each test of a morph-specific difference in flux through an individual pathway of metabolism, or allocation of an individual product of metabolism to a somatic or ovarian compartment, was independent of the other tests. The same was true for all tests of metabolic or allocation trade-offs. Furthermore, all tests were planned a priori contrasts used to investigate specific metabolic or allocation hypotheses. Thus " $P$ " values from individual tests do not require Bonferroni-corrections for experiment-wide error.

\subsection{Statistical analyses}

2.5.1. Morph-specific metabolism of glycine through individual pathways and allocation to individual body compartments

Phenotypic differences between morphs in flux through a single metabolic pathway or allocation to a single body compartment were assessed by ANCOVA (part one of this study). The independent variable in the ANCOVA was DPM (radioactivity) of the metabolic product in whole bodies or in the body compartment in question, on a particular diet. The factors tested were MORPH [LW(f) or SW] and DIET (100\%, $50 \%$, and $25 \%$ ). The covariate was total DPM from glycine incorporated into all metabolites in all body compartments (total incorporated counts; "TC"). Use of this covariate standardizes incorporation of radiolabel into a particular metabolite to total incorporation, which, in turn, eliminates the possibility that a difference between morphs in incorporation of radiolabel into a specific metabolite is simply due to a difference in TC. The overall ANCOVA was followed by tests of morph-differences on each of the three diets.

\subsubsection{Morph-specific metabolic and allocation trade-offs}

Incorporation of radiolabel from glycine into $\mathrm{CO}_{2}$, protein, and various lipid components in ovarian and somatic (whole- 
body minus ovaries) body compartments was measured in all individuals in this study. This allowed proportional metabolism of glycine (metabolic trade-offs; Fig. 1a) and proportional allocation of metabolic products to somatic vs. reproductive body compartments (allocation trade-offs; Fig. 1b) to be quantified in each individual on each of the three diets and to be compared between morphs. Morph-specific trade-offs were tested by ANOVA of these proportions after arcsine transformation (to normalize distributions of proportions) (see Table 3 for calculation of proportions). As in the ANCOVAs, the fac-

Table 1.

Whole-body and ovarian wet mass (mg) of wing morphs of G. firmus

\begin{tabular}{llll}
\hline Age/morph & Whole-body mass & Ovarian mass & Percent \\
\hline C-1 line & & & \\
Day 0 LW(f) & $260 \pm 10.6(7)$ n.s. & $7.6 \pm 0.4(7)$ n.s. & $3.0 \pm 0.2(7)$ n.s. \\
SW & $226 \pm 11.4(6)$ & $7.5 \pm 0.4(6)$ & $3.4 \pm 0.2(6)$ \\
Day 5 LW(f) & $322 \pm 10(22)$ n.s. & $16.9 \pm 4.1(22)^{* * * *}$ & $5.0 \pm 0.9(22)^{* * * *}$ \\
SW & $306 \pm 11(29)$ & $48.0 \pm 4.2(29)$ & $15.1 \pm 1.0(29)$ \\
C-2 line & & & \\
Day-5 LW(f) & $444 \pm 20(8)^{* * * *}$ & $31.1 \pm 6.4(8)^{* * * *}$ & $6.9 \pm 2.1(8)^{* * * *}$ \\
$\quad$ SW & $352 \pm 20(8)$ & $66.4 \pm 6.4(8)$ & $19.5 \pm 2.1(8)$ \\
\hline
\end{tabular}

Values outside of parentheses are means \pm (SEM). Values inside parentheses are sample sizes. n.s. $=$ non significant $(P>0.05), * * *=P<0.001$ by one-way ANOVA (day-0 of the C-1 line and day-5 of the C-2 line), or two-way ANOVA (day 5 of the $\mathrm{C}-1$ line). Percentages were arcsine transformed prior to ANOVA. Day 0 (day of molt to adulthood) crickets and day- 5 crickets of the $\mathrm{C}-2$ line had been raised exclusively on the standard diet, while day- 5 crickets of the $\mathrm{C}-1$ had been raised on three diets starting on day 0 . Sample sizes for day-5 C-1 crickets: $\mathrm{LW}(\mathrm{f})=7,7,8$; $\mathrm{SW}=7,11,11$, for the $100 \%, 50 \%$ and $25 \%$ diets, respectively. For C-1 day-5 crickets, there was no significant interaction between MORPH and DIET for either whole-body mass $(P=0.35)$ or ovarian mass $(P=0.34)$; thus only masses pooled across the three diets are given here. tors tested in ANOVAs of proportions were MORPH [LW(f) and SW], and DIET (100\%, 50\%, and 25\%).

\section{Results}

On the day of molt to adulthood (day 0), ovaries were small and equivalent in both morphs of the $\mathrm{C}-1$ line (Table 1). By contrast, on day 5 , ovaries of this line were 3 times larger in SW females, with reduced flight muscles and reduced wings, compared with LW(f) females with large functional flight muscles and wings (Table 1). The greater increase of ovaries in SW vs. LW(f) females was independent of diet in the $\mathrm{C}-1$ line. That is, the magnitude of increase was statistically equivalent in SW vs. LW(f) females on each of the three diets, and there was no interaction between MORPH and DIET. Crickets of the C-2 line, which were exclusively reared on the standard $(100 \%)$ diet, also exhibited a significantly greater ovarian mass in SW vs. LW(f) females on day 5 of adulthood. Thus, the standard trade-off between masses of ovaries and organs of flight, observed in previous studies of $G$. firmus (Zera, 2005), was observed in the present study.

On the day of molt to adulthood, no differences were observed between the LW(f) and SW morphs of the C-1 line with respect to any aspect of amino acid metabolism, or allocation of end products of metabolism to ovaries or soma (whole body minus ovaries) (Table 2). Similarly, no differences were observed between morphs with respect to tradeoffs in proportional metabolism of glycine or proportional allocation of end products of glycine metabolism to ovarian or somatic organs on this day in C-1 females (data not shown). By contrast, on day 5 of adulthood, large magnitude differences were observed between morphs with respect to most aspects of absolute or proportional metabolism of glycine and allocation of end products of metabolism to the ovaries

\section{Table 2.}

Incorporation of radiolabel from glycine into various metabolites in whole-bodies and somatic and ovarian body compartments of $G$. firmus of the C-1 line on day 0 (day of molt to adulthood)

\begin{tabular}{llcl}
\hline Constitutent & \multicolumn{1}{l}{ Morph } & & Results of ANCOVA \\
\cline { 2 - 4 } & LW(f) & SW & \\
\hline Whole-body (WB) protein & $125,694 \pm 10,789(6)$ & $120,392 \pm 10789(6)$ & n.s. \\
$\quad$ Somatic protein & $123,731 \pm 7797(5)$ & $114,472 \pm 7103(6)$ & n.s. \\
Ovarian protein & $2249 \pm 456(6)$ & $2318 \pm 456(6)$ & n.s. \\
$\mathrm{CO}_{2}$ & $17,532 \pm 2794(5)$ & $16,002 \pm 2545(6)$ & n.s. \\
WB Lipid & $5892 \pm 345(5)$ & $5940 \pm 314(6)$ & n.s. \\
$\quad$ Somatic total lipid & $4817 \pm 264(7)$ & $4820 \pm 285(6)$ & n.s. \\
$\quad$ Ovarian total lipid & $436 \pm 33(6)$ & $466 \pm 33(6)$ & n.s. \\
WB triglyceride & $529 \pm 50(5)$ & $539 \pm 45(6)$ & n.s. \\
WB phospholipid & $5007 \pm 310(5)$ & $5041 \pm 282(6)$ & n.s. \\
\hline
\end{tabular}

Values are mean $( \pm$ SEM) incorporation of radiolabel (DPM) standardized to total incorporated counts (covariate). ANCOVAs performed using total body wet mass alone or with total incorporated counts as the covariate gave essentially identical results as those shown above (data not shown). n.s. $=P>0.4$. 
or soma in C-1 females (Fig. 2 and Fig. 3; Table 3). A similar situation was observed for day- 5 morphs of the C-2 line which were fed exclusively the standard diet (Table 4). Results for the $\mathrm{C}-1$ line will be considered first.

\subsection{Metabolism of glycine through individual metabolic pathways, and allocation to individual body compartments in $\mathrm{C}-1$ crickets}

Radiolabelled carbon from glycine was predominantly converted into protein (ca. 75\% ), followed by $\mathrm{CO}_{2}$ (ca. 20\%), and total lipid (<5\%) (Fig. 2). C-1 SW females converted a greater amount of radiolabelled carbon from glycine into whole-body protein than did LW(f) females. Conversely, $\mathrm{LW}(\mathrm{f})$ females oxidized a greater amount of glycine into $\mathrm{CO}_{2}$, and converted a greater amount of rabiolabled gly- cine into whole body total lipid. The greater amount of total lipid was due to an elevated production of both of the major lipid components, triglyceride and phospholipid.

Diet also had a strong effect on glycine metabolism, influencing the incorporation of radiolabel from glycine into each of the main end products of metabolism (Fig. 2). In each morph, the general trend was increased conversion of glycine into whole-body protein, coupled with decreased oxidation of glycine, and decreased conversion of glycine into lipids from the standard high nutrient $(100 \%)$ diet, to the high carbohydrate diet (50\%), to the low nutrient (25\%) diets. This trend was especially evident in the decreased conversion of radiolabel from glycine into whole-body lipid and triglyceride from $100 \%$ to $25 \%$ diets (Fig. 2).

Although diet had a strong effect on each of the main pathways of glycine metabolism, except in one case, the ef-
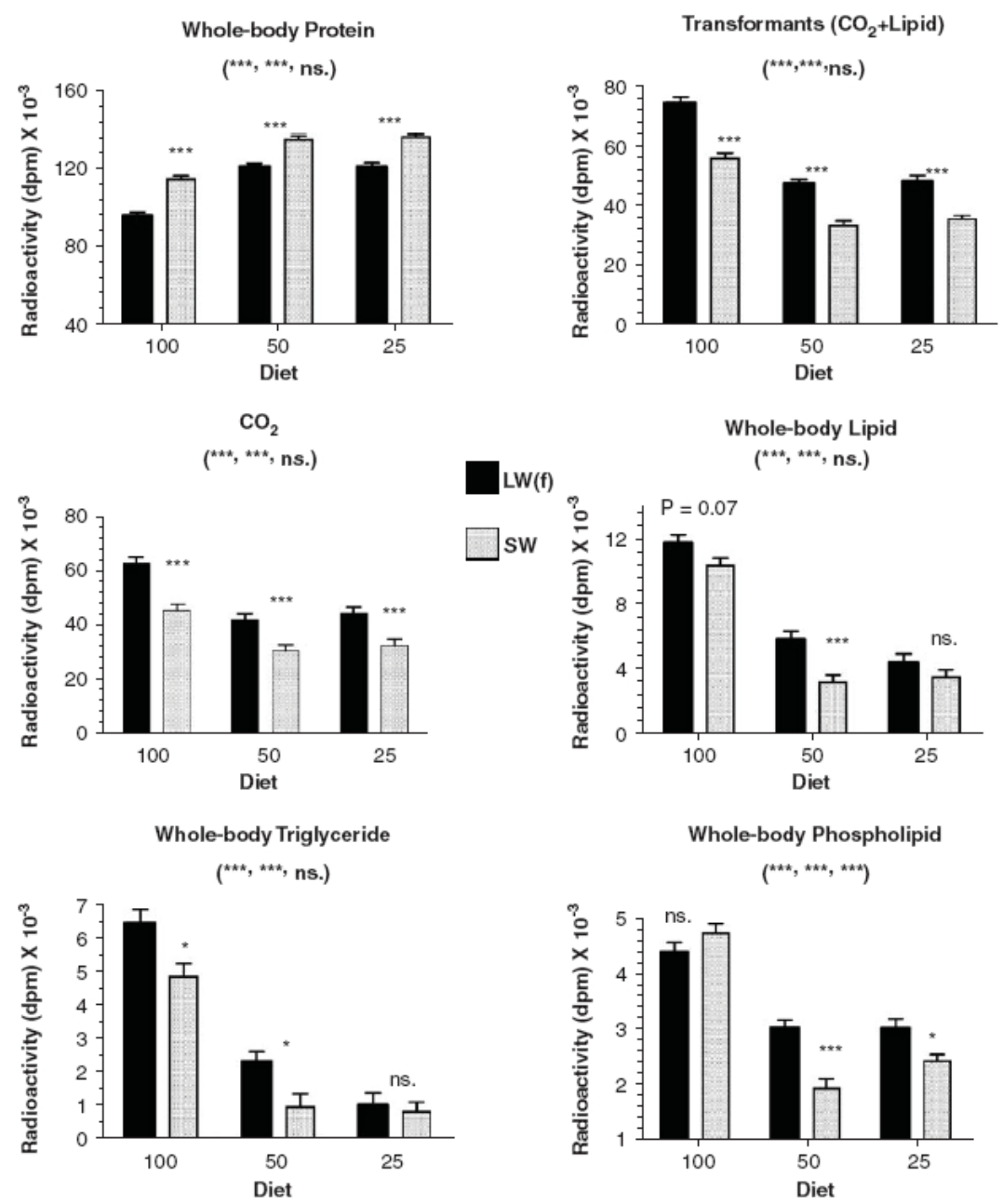

Figure 2. Whole-organism rates of metabolism of glycine into protein, $\mathrm{CO}_{2}$, total lipid, and major lipid components in day-5 LW(f) and $\mathrm{SW}$ morphs of $G$. firmus of the C-1 line fed three diets. Histograms represent mean \pm SEM radiolabel (DPM), derived from injected ${ }^{14} \mathrm{C}$-glycine, that was incorporated into various metabolic end products during the $5 \mathrm{~h}$ incubation period. Sample sizes are given in Table 3. Incorporated DPM were standardized to total incorporation by ANCOVA (see Methods). These standardized DPM are virtually identical to unstandardized DPM. The first symbol within the parentheses denotes the overall effect of morph (pooled across diets), the second symbol denotes the overall effect of diet, and the third symbol denotes the interaction between morph and diet (i.e. dependence of morph-differences on specific diets). Symbols above histograms refer to analyses of morph-differences within individual diet treatments $(* * *=P<0.005 ; *=P<0.05$; n.s. $=$ non-significant $)$. 

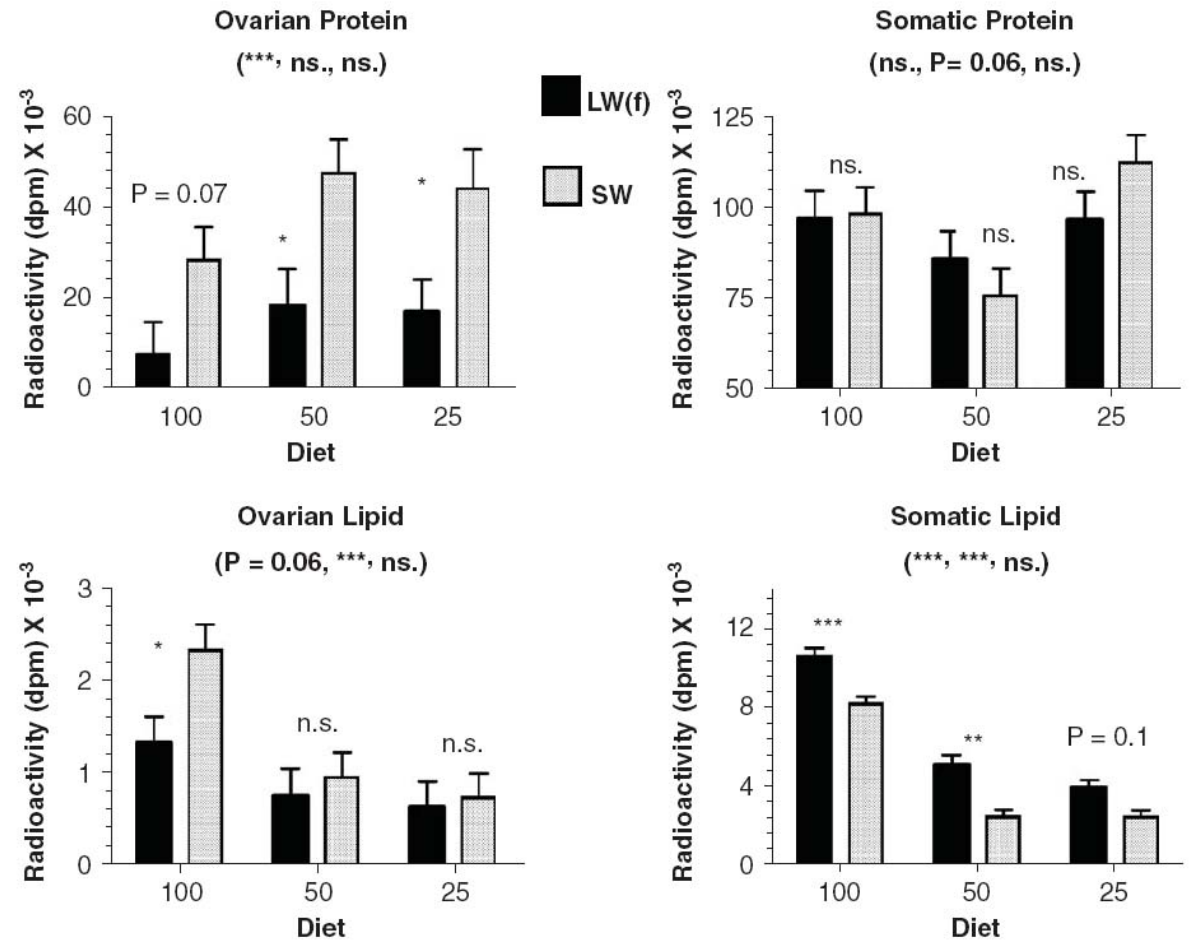

Figure 3. Incorporation of radiolabel from glycine into various end products of metabolism allocated to ovarian and somatic (whole body minus ovaries) body compartments during the $5 \mathrm{~h}$ incubation period. Symbols and sample sizes are the same as those in Fig. 2.

Table 3.

Proportional diversion (trade-off) of radiolabel from ${ }^{14} \mathrm{C}$-glycine to various metabolites in whole-bodies, and various body compartments in flight-capable $[\mathrm{LW}(\mathrm{f})]$ or flightless (SW) G. firmus from the C-1 line on day 5 of adulthood

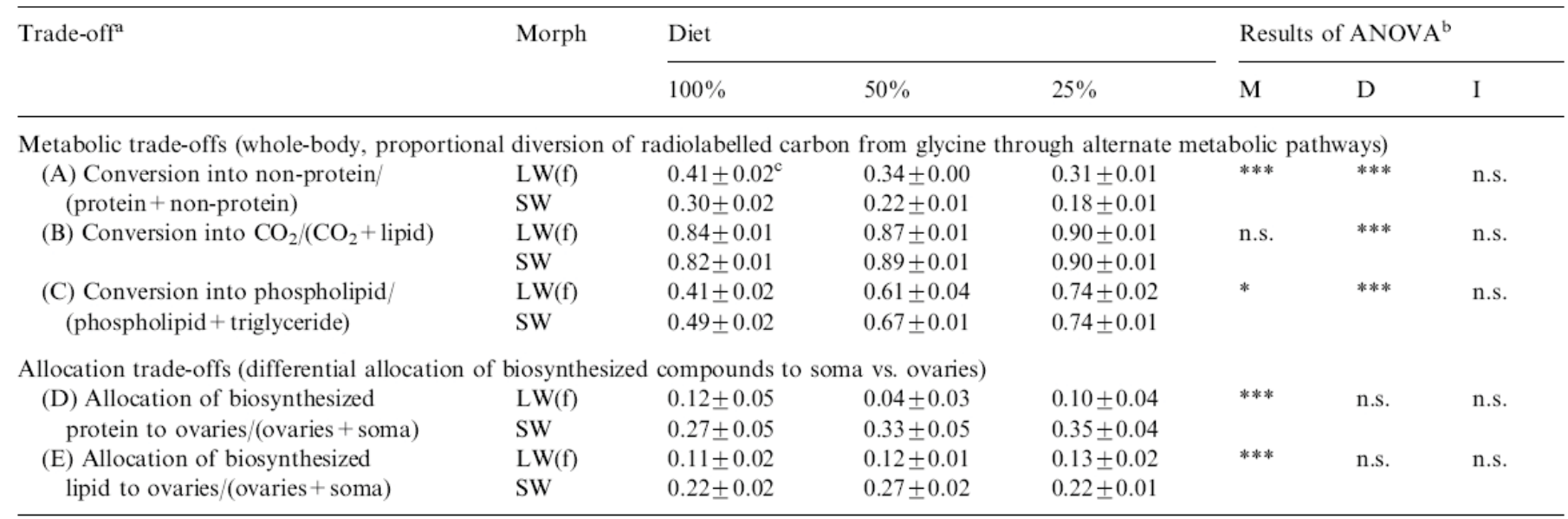

${ }^{a}$ Letters refer to trade-offs illustrated in Fig. 1.

b The first symbol denotes the overall effect of morph ("M"; data pooled across diets) on the proportion of radiolabel diverted into alternate products or body compartments (morph-specific trade-off); the second symbol denotes the overall effect of diet ("D") on the diversion of radiolabel into alternate products or body compartments; the third symbol denotes the interaction between morph and diet ("I"; dependence of morph-differences on specific diets) $(* * *=P<0.005 ; *=P<0.05$; n.s. $=$ non-significant); Note; the same results are obtained in statistical analyses irrespective of which of the two traits is chosen as the numerator.

${ }^{c}$ Values are mean $( \pm$ SEM) proportion of radiolabel diverted through one of two linked pathways, or into one of two body compartments. Means are based on $7-8$ individuals [LW(f) $100 \%$ or $25 \%$, SW $100 \%$ or $50 \%$ ] or $11-14$ [LW(f) $50 \%$, or SW $25 \%$ ].

fect of diet was similar in both morphs. That is, MORPH $\times$ DIET interactions were non-significant in all cases except phospholipid production from glycine (Fig. 2). This aspect of glycine metabolism did not differ between the morphs on the standard (100\%) diet, but was significantly higher in LW(f) vs. SW females on the $50 \%$ and $25 \%$ diets. 
Table 4.

Incorporation of radiolabel (DPM) from ${ }^{14} \mathrm{C}$-glycine into various chemical constituents and body compartments in day-5 G. firmus of the C-2 line fed the standard $(100 \%)$ diet

(1) Absohate incorporation of radiolabel from glycine into a single whole-body metabolite, or into an individual body compartment

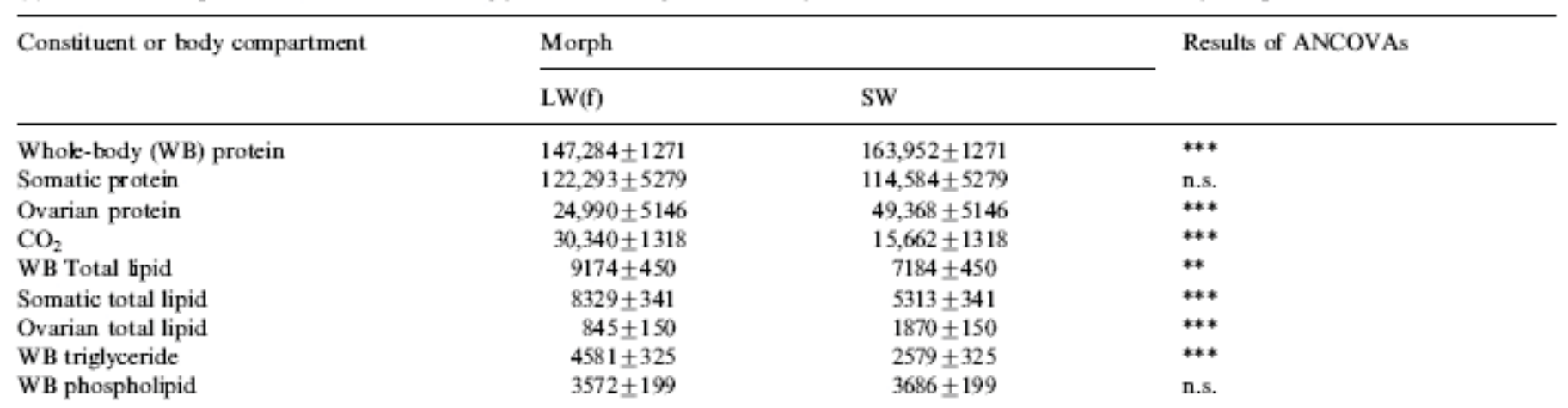

(2) Proportional incorporation (trade-off) of radiolabel from glycine into alternate metabolites, or into ovaries vs. somatic body compartments

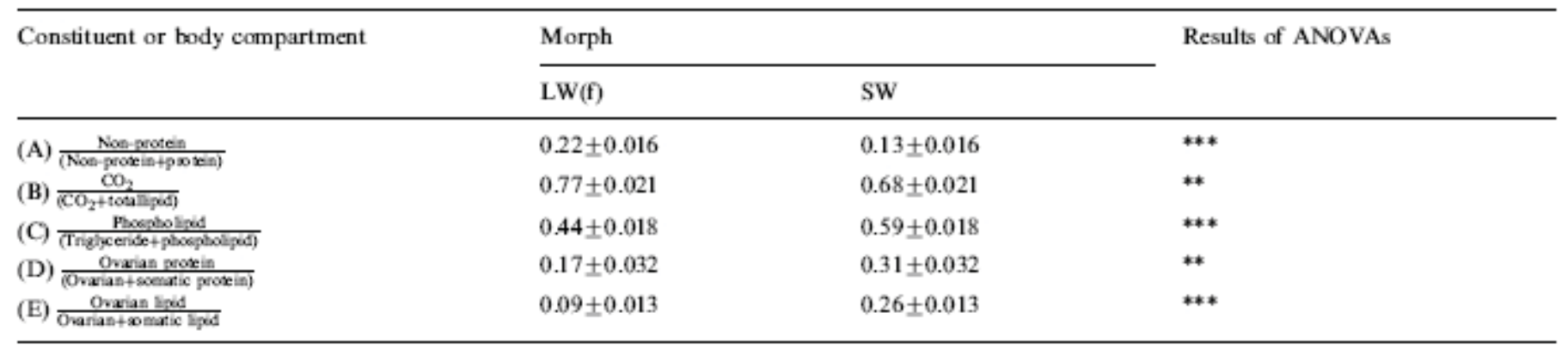

Means are based on eight individuals; for ANCOVAs, total counts ("TC") was the covariate (see Methods); ANOVAs were performed on aresine transformed proportions to normalize the data; ${ }^{* * *}=P<0.005 ;^{* *}=P<0.01{ }^{*}=P<0.05$.

With respect to allocation of biosynthesized products, SW females allocated significantly more biosynthesized protein or lipid $(P=0.06)$ to the ovaries than did LW(f) females (Fig. 3). The amount of biosynthesized protein in the soma did not differ between the morphs, while LW(f) females allocated a greater amount of biosynthesized lipid to the soma than did SW females. Diet had only a minor effect on protein allocation to somatic or ovarian body compartments. By contrast, both morphs allocated a substantially reduced amount of biosynthesized lipid to the soma or ovaries on the $50 \%$ and $25 \%$ diets (Fig. 3). As was the case for whole-organism production of end products of glycine metabolism (Fig. 2), MORPH $\times$ DIET interactions were not significant for allocation of protein or lipid produced from glycine to the soma or ovaries (Fig. 3). Thus, although allocation of end products of glycine metabolism was, in general, strongly affected by diet, the effect was similar on both morphs.

\subsection{Metabolic and allocation trade-offs in the C-1 line}

Data described above refer to incorporation of radiolabel from glycine into various individual end products of metab- olism in individual (somatic or ovarian) body compartments. When proportional production of products from linked pathways of metabolism (metabolic trade-offs), or proportional allocation of a single product to different body compartments (allocation trade-offs) was considered, a number of large-magnitude trade-offs was documented (Table 3). In no case was a MORPH $\times$ DIET interaction observed, and thus morph-specific trade-offs occurred to a similar degree across diets. Finally, diet, independent of morph, strongly affected metabolic but not allocation trade-offs.

SW females converted a proportionately greater amount of radiolabelled carbon from glycine into protein than did LW(f) females, while LW(f) females diverted a greater proportion of carbon from glycine through the Krebs Cycle to $\mathrm{CO}_{2}$ plus lipid (Trade-off "A"; Table 3; Fig. 1a). Compared with LW(f) females, SW females biosynthesized a proportionately greater amount of phospholipid as opposed to triglyceride (Trade-off "C"; Table 3, Fig. 1a). Finally, although $\mathrm{LW}(\mathrm{f})$ females produced a proportionately greater amount of both $\mathrm{CO}_{2}$ and total lipid from glycine than $\mathrm{SW}$ females, the proportional production of these two products did not differ between the morphs. In other words, trade- 
off "B" (Table 3; Fig. 1a) was not significant. Both morphs converted a proportionately greater amount of carbon from glycine into protein vs. non-protein products, into $\mathrm{CO}_{2}$ vs. lipid, and into phospholipid vs. triglyceride on non-standard $(25 \%$ and $50 \%)$, compared with the standard diet.

With respect to allocation of metabolic products to ovaries vs. soma, SW females allocated a proportionately greater amount of protein and lipid produced from glycine to the ovaries, while LW(f) females diverted a proportionately greater amount of these products to the soma. DIET had no effect on these two allocation trade-offs (Table 3).

\subsection{Absolute and relative metabolism of glycine and al- location of end products to the ovaries vs. soma for $\mathrm{C}-2$ crickets raised on the standard diet}

Crickets of the other control line (C-2), which had been raised exclusively on the standard diet, exhibited similar patterns of glycine metabolism and allocation of end products to somatic vs. ovarian compartments as crickets of the $\mathrm{C}-1$ line fed the standard diet (Table 4). As was the case for the C-1 line, SW females of the $\mathrm{C}-2$ line incorporated a significantly greater amount of radiolabelled carbon from ${ }^{14} \mathrm{C}$-glycine into wholebody protein, ovarian protein, and ovarian total lipid compared with LW(f) females. Similarly, LW(f) females incorporated a significantly greater amount of radiolabel from glycine into $\mathrm{CO}_{2}$, whole-body total lipid, somatic total lipid and triglyceride. Finally, except for the significant morph-specific trade-off in the diversion of radiolabel into $\mathrm{CO}_{2}$ vs. lipid (non-significant in C-1 crickets), all significant trade-offs in C-2 crickets were observed in $\mathrm{C}-1$ crickets (Table 4).

\section{Discussion}

Results obtained in the present study relate to two important topics: the biochemical basis of morph-specialization in dispersal polymorphic insects, and the more general issue of the biochemical basis of life history trade-offs. Adaptations for flight vs. reproduction in dispersal polymorphic insect species have primarily been studied from physiological or morphological perspectives. Relatively little information is available on the biochemical basis of these adaptations, especially with respect to in vivo aspects of intermediary metabolism. Similarly, there is a paucity of biochemical information for the more general topic of life history trade-offs (Zera and Harshman, 2001; Zhao and Zera, 2002; Zera, 2005). Yet the differential diversion of nutrients through alternate pathways of metabolism is expected to be a key component in life history trade-offs and morph specializations for dispersal vs. flight. The present and recent studies in G. firmus (Zera and Harshman 2001; Zhao and Zera 2002; Zera, 2005; Zera and Zhao, 2006) constitute the first direct demonstrations and estimations of the magnitudes of in vivo trade-offs in flux through pathways of intermediary metabolism that underlie within-species life history trade-offs, such as specializations for flight vs. reproduction in dispersal polymorphic insects.

\subsection{Morph-specific trade-offs in amino acid metabolism}

One of the most important findings of the present study was the ubiquity of large-magnitude trade-offs of internal resources in control lines of G. firmus (Fig. 2; Table 3). LW(f) and SW morphs typically differed by about $40-50 \%$ in the proportional allocation of radiolabelled carbon from glycine into alternate products of metabolism or into somatic vs. reproductive organs. Importantly, these trade-offs occurred during but not prior to the period of adulthood when the dramatic trade-off occurs between ovarian growth and triglyceride accumulation in the morphs (Zera and Larsen 2001), and were observed when crickets were fed a variety of diets. These results, combined with data from other recent studies on lipid and amino acid metabolism in artificially selected lines of G. firmus (see below), strongly suggest that largescale modifications of multiple, interacting pathways of intermediary metabolism are key components of the specialization for reproduction vs. flight-capability in G. firmus.

As expected, SW females diverted a greater amount of ${ }^{14} \mathrm{C}$-glycine to biosynthesis of ovarian protein at the expense of somatic protein on each diet (Fig. 3). Elevated biosynthesis of ovarian proteins such as vitellogenin is likely a major factor responsible for the greater ovarian growth in the SW morph, which occurs primarily by accumulation of protein into eggs. The higher rate of total protein biosynthesis from glycine in SW vs. LW(f) morphs (Fig. 2) suggests that total protein biosynthesis is not equivalent in the morphs, and that the enhanced reproductive output of the SW morph requires more protein biosynthesis than does flight capability in the LW morph. To our knowledge, this is the first estimate of the relative costs of these two important organismal functions, in terms of protein biosynthesis, in any organism.

It is likely that other functionally important trade-offs exist between morphs involving amino acid metabolism, which were not measured in the present study. For example, a greater proportion of somatic protein biosynthesis was likely devoted to the production of flight muscle and fat body proteins in LW(f) vs. SW females, because these two organs are considerably larger in the LW(f) morph (Zera et al. 1997; Zera and Zhao 2003a). An important topic for future research is the contribution of these organ-specific differences in amino acid metabolism to the overall flight-fecundity trade-off.

Another important finding of the present study was the substantially greater oxidation of glycine by LW(f) vs. SW females on each of three diets (Fig. 2; Table 3 and Table 4). This observation is noteworthy in light of the reverse situation for morph-specific oxidation of fatty acids, which is $100 \%$ greater in SW than in $\mathrm{LW}(\mathrm{f})$ females on day 5 of 
adulthood (Zera and Zhao, 2003b; Zera, 2005). The present and previous studies collectively demonstrate remarkable morph-specific complementary interactions between amino acid and lipid metabolism that result in the morph-specific sparing vs. oxidation of specific nutrients. Increased oxidation of amino acids for energy in LW(f) females appears to preferentially spare fatty acids, allowing their enhanced conversion into triglyceride, the main flight fuel of this species. Conversely, increased oxidation of fatty acid by the SW morph appears to preferentially spare amino acids for enhanced conversion to ovarian protein. To our knowledge these data together with analogous information on genetic interactions between lipid and amino acid metabolism (Zera and Zhao, 2006; see below) are the first detailed information on interactions between different pathways of intermediary metabolism that underlie adaptations to different life histories, either within or between species. These data also constitute one of the most striking cases of adaptive divergence in intermediary metabolism among phenotypes within a species of which we are aware. These key metabolic differences could only have been identified by detailed in vivo studies of metabolism. Because the respiratory quotients of amino acid and lipid are approximately equivalent (Withers, 1992), differences between the morphs in lipid vs. amino acid oxidation would have gone undetected if metabolic differences between morphs had only been compared using whole-organ respirometry, a common approach in functional studies of life history trade-offs (Zera and Harshman, 2001).

LW(f) females also diverted a greater amount of amino acid through the Krebs Cycle into lipid, compared with SW females (Fig. 2, Table 3 and Table 4). This greater diversion likely reflects the higher demand for triglyceride flight fuel (Zera et al., 1999) in the LW(f) morph (note: morphs did not fly during the study; the differential production of triglyceride solely reflects the preparation for flight). Indeed LW(f) females preferentially converted radiolabelled carbon from glycine into triglyceride as opposed to phospholipid (Table 3 and Table 4, Fig. 2); phospholipid is a structural lipid not used as a flight fuel. Previous studies (Zera and Zhao, 2003a,b; Zhao and Zera, 2002; Zera, 2005) have identified three important metabolic modifications that underlie the enhanced accumulation of triglyceride flight fuel (Table 5): (a) increased de novo biosynthesis of fatty acids, (b) greater proportional diversion of fatty acids into triglyceride vs. phospholipid, and (c) reduced oxidation of lipid. The present study has identified a fourth metabolic modification: increased conversion of amino acids into triglyceride.

The higher rate of amino acid metabolism through the Krebs Cycle by LW(f) lines, observed in the present in vivo study (Fig. 2, Table 3 and Table 4), is supported by independent evidence obtained in a previous in vitro enzymological study (Zera and Zhao 2003a). In that study, LW(f) lines of $G$. firmus exhibited significantly higher in vitro activities of alanine and aspartate aminotransferases, enzymes which convert amino acids into Krebs Cycle intermediates. The existence of elevated activities of multiple aminotransferases in LW(f) lines, which act on different classes of amino acids, also suggests that enhanced diversion of amino acids through the Krebs Cycle likely occurs for amino acids in general, not only for glycine.

Variation in the allocation of nutrients to life history traits can result from variation in the intake of external re-

Table 5.

Summary of differences in amino acid and lipid metabolism between flight-capable and flightless morphs of G. firmus

\begin{tabular}{lll}
\hline Aspect of metabolism & $\begin{array}{l}\text { Flight capable [LW(f)] morph } \\
\text { relative to flightless (SW) morph }\end{array}$ & References \\
\hline Absolute biosynthesis of whole-body (WB) lipid & Higher & A, B \\
Absolute biosynthesis of WB triglyceride & Higher & A \\
Absolute biosynthesis of WB phospholipid & Lower & A \\
Relative biosynthesis of triglyceride vs. phospholipid & Higher & A \\
Oxidation of fatty acid & Lower & C \\
Relative utilization of fatty acid for biosynthesis & Higher & C \\
vs. oxidation & Higher & B, D \\
Specific activities of lipogenic enzymes & Higher & E \\
Conversion of amino acids into WB lipid & Higher & Higher \\
Oxidation of amino acid & Lower & B, D \\
Activity of transaminases & & E \\
Biosynthesis of ovarian protein from amino acids & Higher & A, E \\
Allocation of biosynthesized lipid to soma vs. ovaries & Lower & A, E \\
Triglyceride & Equivalent & F \\
Phospholipid & &
\end{tabular}

Values are for individuals reared on the "standard" diet (See Zera and Larsen, 2001 for diet composition). Flight capable=long-winged individuals with large pink (functional) flight muscles; flightless=short-winged individuals with underdeveloped flight muscles.

References: A=Zhao and Zera, 2002; B=Zhao and Zera, 2001; C=Zera and Zhao, 2003a; D=Zera and Zhao, 2003b; E=Present Study and Zera and Zhao (2006); F=Zera and Brink, 2000. 
sources as well as by the differential biosynthesis of internal nutrients (Boggs, 1997a and Boggs, 1997b; O'Brien et al., 2002 and O'Brien et al., 2005; Zera and Harshman, 2001) (see below). On the standard diet, LW(f) and SW lines only differ to a small degree $(<10 \%)$ in total food consumption or assimilation of total nutrients, protein, lipid, or carbohydrate from ingested food (A. J. Zera, unpublished data). This situation is similar to flightless and flight-capable morphs in other Gryllus species, which do not differ significantly in nutrient consumption or assimilation (Mole and Zera, 1993; Zera and Harshman, 2001; Zera et al., 1998). Thus, the primary mechanism by which various chemical constituents such as protein and lipid are differentially accumulated and allocated to reproductive vs. somatic functions is by morph-specific metabolic conversions of nutrients following assimilation from the diet. Wing polymorphism in G. firmus is one of the only experimental models for which quantitative assessments have been made regarding the relative importance of variation in nutrient acquisition vs. variation in internal nutrient biosynthesis to a life history trade-off. Surprisingly, very limited data are available on nutrient acquisition in most experimental models used in physiological studies of life history trade-offs (Zera and Harshman 2001), a notable exception being the studies of Boggs and co-workers ((Boggs, 1997a and Boggs, 1997b; O'Brien et al., 2002 and O'Brien et al., 2005); see Zera and Zhao (2006) for an extensive discussion for work by Boggs and co-workers as it relates to morph-specific amino acid metabolism in Gryllus.

\subsection{Comparison between phenotypic studies of control lines vs. genetic studies of selected lines}

Phenotypic differences in amino acid metabolism between LW(f) and SW morphs from unselected control lines report- ed in the present study were very similar to genetic differences in amino acid metabolism between morphs from selected lines of the same artificial selection experiment reported in a companion study (Zera and Zhao, 2006; Table 6). For each trade-off in amino acid metabolism, direction and magnitude of the difference between LW(f) and SW morphs were similar in these two studies (Table 6). For example, the median average difference between morphs (difference between morphs/mean of morphs for each trade-off averaged over all trade-offs) was $44 \%$ and $47 \%$, respectively, for morphs from control vs. selected lines (Table 6). For both control and selected lines, morphs differed to a much greater degree for trade-offs " $D$ " and "E" than trade-offs " $A$ " and "C". These parallel phenotypic and genetic differences between morphs in control and selected lines of G. firmus bear on an important empirical issue in the analysis of life history trade-offs.

The extent to which genetic vs. phenotypic analyses are appropriate methods to study life history evolution is a subject of debate (Rose et al., 1996; Zera and Harshman, 2001). Genetic analyses are especially relevant for evolutionary investigations of life histories, but they also are much more labor intensive than phenotypic analyses (but which cannot estimate the proportion of phenotypic variation due to genetic vs. environmental causes). Genetic analyses of continuous traits (e.g. most biochemical and physiological traits) either require multigenerational artificial selection or single generation breeding designs (e.g. half sib analyses), both of which are very labor intensive because of the large sample sizes required. If conclusions derived from phenotypic analyses of unselected control lines are similar to those derived from genetic analyses of artificially selected lines, then phenotypic analyses would be advantageous, because they are much easier to perform, and because it is difficult to perform genetic crosses on many organisms. Few studies have directly compared results obtained from genetic analyses of selected lines

Table 6.

Proportional conversion (trade-offs) of ${ }^{14} \mathrm{C}$ from glycine into alternate metabolites or allocation into alternate compartments in control or selected lines of G. firmus fed the standard (100\%) diet

\begin{tabular}{|c|c|c|c|c|c|c|}
\hline \multirow[t]{2}{*}{ Trade-off } & \multirow[t]{2}{*}{ Morph } & \multicolumn{3}{|l|}{ Selected lines } & \multicolumn{2}{|c|}{ Control lines } \\
\hline & & Bk-1 & Bk-1 & Bk-3 & $C-1$ & $\mathrm{C}-2$ \\
\hline \multirow{2}{*}{ (A) $\frac{\text { Non-protein }}{\text { (Non-protein }+ \text { protein) }}$} & $\mathrm{LW}(\mathrm{f})$ & $0.34 \pm 0.01^{\mathrm{a}}$ & $0.22 \pm 0.03$ & $0.15 \pm 0.02$ & $0.41 \pm 0.02$ & $0.22 \pm 0.02$ \\
\hline & SW & $0.26 \pm 0.02$ & $0.14 \pm 0.02$ & $0.09 \pm 0.01$ & $0.30 \pm 0.02$ & $0.13 \pm 0.02$ \\
\hline \multirow{2}{*}{ (B) $\frac{\mathrm{CO}_{2}}{\left(\mathrm{CO}_{2}+\text { lipid }\right)}$} & LW(f) & $0.82 \pm 0.02$ & $0.74 \pm 0.03$ & $0.74 \pm 0.03$ & $0.84 \pm 0.01$ & $0.77 \pm 0.02$ \\
\hline & SW & $0.83 \pm 0.03$ & $0.74 \pm 0.03$ & $0.44 \pm 0.04$ & $0.82 \pm 0.01$ & $0.68 \pm 0.02$ \\
\hline \multirow{2}{*}{ (C) $\frac{\text { Phospholipid }}{\text { (Phosphlipid+triglyceride) }}$} & $\mathrm{LW}(\mathrm{f})$ & $0.41 \pm 0.02$ & $0.48 \pm 0.02$ & $0.52 \pm 0.03$ & $0.41 \pm 0.02$ & $0.44 \pm 0.02$ \\
\hline & SW & $0.53 \pm 0.02$ & $0.54 \pm 0.01$ & $0.63 \pm 0.02$ & $0.49 \pm 0.02$ & $0.59 \pm 0.02$ \\
\hline \multirow{2}{*}{ (D) $\frac{\text { Ovarian protein }}{\text { (Ovarian+somatic protein) }}$} & $\mathrm{LW}(\mathrm{f})$ & $0.14 \pm 0.02$ & $0.12 \pm 0.01$ & $0.22 \pm 0.02$ & $0.12 \pm 0.05$ & $0.17 \pm 0.03$ \\
\hline & SW & $0.31 \pm 0.01$ & $0.32 \pm 0.02$ & $0.34 \pm 0.01$ & $0.27 \pm 0.02$ & $0.31 \pm 0.03$ \\
\hline \multirow{2}{*}{ (E) $\frac{\text { Ovarian lipid }}{\text { (Ovarian+somatic lipid) }}$} & $\mathrm{LW}(\mathrm{f})$ & $0.10 \pm 0.01^{\mathrm{b}}$ & $0.14 \pm 0.02$ & $0.14 \pm 0.02$ & $0.11 \pm 0.02$ & $0.09 \pm 0.01$ \\
\hline & SW & $0.29 \pm 0.02$ & $0.54 \pm 0.03$ & $0.50 \pm 0.01$ & $0.22 \pm 0.02$ & $0.26 \pm 0.01$ \\
\hline
\end{tabular}

${ }^{\text {a }}$ Data are mean ( \pm SEM) values for selected lines [data from Zera and Zhao (2006)] or from control lines (Table 3 and Table 4)]. Only data for crickets raised on the standard diet are given here. Means for selected lines were based on assays of 6-9 individuals.

b For selected lines "lipid"=triglyceride (total lipid was not measured), while for control line "lipid"=total lipid. 
with phenotypic analyses obtained from control lines of the same artificial selection study (Leroi et al., 1994; Zera and Bottsford, 2001; Zera and Harshman, 2001). The similar results obtained from the present phenotypic and companion genetic studies (Table 6) indicate that the less labor intensive phenotypic analyses appear to be useful surrogates for the much more laborious genetic analyses with respect to identifying evolutionary important phenotypic variation in amino acid metabolism underlying life history trade-offs in G. firmus. These results are similar to ecdysteroid titers, which also exhibit parallel differences between morphs from control vs. between artificially selected lines (Zera and Bottsford, 2001).

It is important to note two important limitations of our biochemical studies of life history trade-offs in Gryllus. First, all biochemical studies of Gryllus have been undertaken in long-term laboratory populations that had been maintained for several years after initiation from field-collected individuals. We currently have no information on biochemical differences between morphs in field populations measured under field conditions. This limitation also applies to the vast majority of studies of dispersal polymorphism and life history evolution in insects, which also have typically been undertaken on long-term laboratory populations. Biochemical adaptations different from those in field populations can evolve in long-term laboratory populations (Gibbs, 1999; Harshman and Hoffmann, 2000). Thus, an important topic for future research is the extent to which biochemical specializations for dispersal and reproduction identified in laboratory populations also occur in individuals in the field. The second limitation is that all biochemical studies on intermediary metabolism in dispersal polymorphic species thusfar have been undertaken in a restricted set of species: those of the genus Gryllus. The extent to which parallel morph differences occur in other wing-polymorphic species is unknown and is also an important topic for future studies.

Data obtained in the present study extend results obtained in the genetic analysis of Zera and Zhao (2006) in several important ways. First, unlike the study of Zera and Zhao (2006) the present study investigated morph differences in amino acid metabolism both during the period of adulthood (day 5) when the trade-off between ovarian growth and triglyceride accumulation occurs (day 5; Fig. 2 and Fig. 3 ; Table 3), as well as prior to that time (day 0; Table 2). The occurrence of morph-differences in amino acid metabolism on day 5 but not on day 0 strengthens the argument that the biochemical differences between morphs contribute to the trade-off between ovarian growth and preparation for flight (i.e. the biochemical differences underlie the life history trade-off). Second, the genetic study of Zera and Zhao (2006) did not investigate the effect of diet on morph differences in amino acid metabolism. In the present study, in nearly all cases, the magnitude of morph-differences in amino acid metabolism were independent of specific diets that are known to have strong effects on morph physiology, and amino acid and lipid metabolism. Thus morph-differences are robust, and are consistently manifest under a wide range of environmental conditions. In many cases (e.g. studies in Drosophila), life history variation and trade-offs are sensitive to environmental conditions and sometimes are manifest sporadically (Rose et al., 1996). Importantly, the failure to observe MORPH $\times$ DIET interactions in G. firmus was not due to an absence of an effect of diet on metabolic characteristics. Diet typically had a strong effect on nearly all aspects of amino acid metabolism, but the effect was similar in both morphs (Fig. 2 and Fig. 3; Table 3 and Table 4). The absence of MORH $\times$ DIET interactions for amino acid metabolism in the present study was similar to the absence of MORPH $\times$ DIET interactions observed in previous studies of lipid oxidation (Zera and Zhao, 2003b), and lipid biosynthesis in G. firmus (Zhao and Zera, 2002). By contrast, strong MORPH $\times$ DIET interactions were observed in studies of lipogenic enzyme activities (Zera and Zhao, 2003a). The significance of the absence of MORPH $\times$ DIET interactions in in vivo studies of lipid and amino acid metabolism coupled with the present of MORPH $\times$ DIET interactions in in vitro studies of lipogenic enzyme activities is unclear at present.

The final point to be made is that the present and previous studies (Zera and Bottsford, 2001; Zera and Cisper, 2001; Zera et al., 1998 and Zera et al., 1997; Zera and Zhao, 2003a and Zera and Zhao, 2003b; Zera and Zhao, 2004 and Zera and Zhao, 2006; Zhao and Zera, 2002) illustrate the exceptional power of wing polymorphism in $G$. firmus as an experimental model to investigate functional aspects of the trade-off between dispersal and reproduction. Differences between LW(f) and SW morphs of G. firmus in aspects of dispersal and reproduction are of large magnitude, are as great or greater than differences between species, and exist over a wide range of environmental conditions. These dramatic, discrete differences in life history, in turn, have provided the material to demonstrate large-magnitude differences in intermediary metabolism and physiology that contribute significantly to life history trade-offs (Zera and Larsen, 2001; Zera and Zhao, 2003a and Zera and Zhao, 2003b; Zera, 2005; Zhao and Zera, 2002) (Fig. 2 and Fig. 3; Table 3, Table 4 and Table 5).

\section{Acknowledgments}

This work was supported by a grants from the National Science Foundation (IBN 9808249 and IBN-0516973) to A.J.Z.

\section{References}

Beenakkers, A.M.T., Van der Horst, D.J., Van Marrewijk, W.J.A., 1985. Biochemical processes directed to flight muscle metabolism. In: Kerkut, G.A., Gilbert, L.I. (Eds.), Comprehensive Insect Physiology, Biochemistry and Pharmacology, vol. 10. Pergamon, Oxford, pp. 451-486.

Boggs, C.L., 1992. Resource allocation: exploring connections between foraging and life history. Functional Ecology 6, 508-518.

Boggs, C.L., 1997a. Dynamics of reproductive allocation from juvenile and 
adult feeding: radiotracer studies. Ecology 78, 192-202.

Boggs, C.L., 1997b. Reproductive allocation from reserves and income in butterfly species with differing adult diets. Ecology 78, 181-191.

Candy, D.J., 1985. Intermediary metabolism. In: Kerkut, G.A., Gilbert, L.I. (Eds.), Comprehensive Insect Physiology Biochemistry and Pharmacology. Pergamon, Oxford, pp. 1-41.

Clements, A.N., 1959. Studies on the metabolism of locust fat body. Journal of Experimental Biology 36, 665-675.

Dingle, H., 1996. Migration: the Biology of Life on the Move. Oxford University Press, Oxford.

Gibbs, A.G., 1999. Laboratory selection for the comparative physiologist. Journal of Experimental Biology 202, 2709-2718.

Harrison, R.G., 1980. Dispersal polymorphisms in insects. Annual Review of Ecology and Systematics 11, 95-118.

Harshman, L.G., Hoffmann, A.A., 2000. Laboratory selection experiments using Drosophila: what do they really tell us? Trends in Ecology and Evolution $15,32-36$.

Kon, R.T., Monroe, R.E., 1971. Utilization of dietary amino acids in lipid synthesis by aseptically reared Musca domestica. Annals of the Entomological Society of America 64, 247-250.

Leroi, A.M., Sung, B.K., Rose, M.R., 1994. The evaluation of phenotypic life history trade-offs using Drosophila melanogaster. American Naturalist $144,661-676$.

Mole, S., Zera, A.J., 1993. Differential allocation of resources underlies the dispersal-reproduction trade-off in the wing-dimorphic cricket, Gryllus rubens. Oecologia 93, 121-127.

O’Brien, D.M., Marilyn, M.L., Boggs, C.L., 2002. Renewable and nonrenewable resources: amino acid turnover and allocation to reproduction. Proceedings of the National Academy of Sciences USA 99, 4413-4418.

O'Brien, D.M., Boggs, C.L., Fogel, M.L., 2005. The amino acids used in reproduction by butterflies: A comparative study of dietary sources using compound-specific stable isotopes ratios analysis. Physiological and Biochemical Zoology 78, 819-827.

Roff, D.A., 1986. The evolution of wing dimorphism in insects. Evolution 40, $1009-1020$

Roff, D.A., 2002. Life history evolution. Sinauer, Sunderland.

Rose, M.A., Nusbaum, T.J., Chippendale, A.K., 1996. Laboratory evolution: The experimental wonderland and the Cheshire cat syndrome. In: Rose, M.R., Lauder, G.V. (Eds.), Adaptation. Academic Press, San Diego.

Socha, R., 1993. Pyrrhocorris apterus (Heteroptera)-an experimental model species: a review. European Journal of Entomology 101, 539-545.

Socha, R., Kodrik, D., 1999. Differences in adipokinetic responses of Pyrrhocoris apterus (Heteroptera) in relation to wing dimorphism and diapause. Physiological Entomology 24, 278-284.

Stryer, L., 1988. Biochemistry. W.H. Freeman and Co., New York.

Tanaka, S., 1994. Endocrine control of ovarian development and flight muscle histolysis in a wing dimorphic cricket, Modicogryllus confirmatus. Journal of Insect Physiology 40, 483-490.

Veazy, J.N., Kay, C.A.R., Walker, T.J., Whitcomb, W.H., 1976. Seasonal abundance, sex ratio, and macroptery of field crickets in northern Florida. Annals of the Entomological Society of America 69, 374-380.

Withers, P.C., 1992. Comparative Animal Physiology. Saunders College, Forth Worth.

Zera, A.J., 2004. The endocrine regulation of wing polymorphism: State of the art, recent surprises, and future directions. Integrative and Comparative Biology 43, 607-616.

Zera, A.J., 2005. Intermediary metabolism and life history trade-offs: Lipid metabolism in lines of the wing-polymoprhic cricket, Gryllus firmus,

selected for flight capability vs. early age reproduction. Integrative and Comparative Biology 45, 511-524.

Zera, A.J., 2006. Wing polymorphism in Gryllus (Orthoptera: Gryllidae): Proximate endocrine, energetic and biochemical mechanisms underlying morph specialization for flight vs. reproduction. In: Whitman, D., Ananthakrishnan, T. (Eds.), Phenotypic Plasticity in Insects: Mechanisms and Consequences. Science Publishers, Plymouth, pp. 441-483.

Zera, A., Zhao, Z., 2006. Intermediary metabolism and life history trade-offs: Differential metabolism of amino acids underlies the dispersal-reproduction trade-off in a wing-polymorphic cricket. American Naturalist, 167 (6).

Zera, A.J., Brink, T., 2000. Nutrient assimilation and utilization by wing and flight muscle morphs of the cricket Gryllus firmus: implications for the trade-off between flight capability and reproduction. Journal of Insect Physiology 46, 1207-1218.

Zera, A.J., Bottsford, J., 2001. The endocrine-genetic basis of life-history variation: Relationship between the ecdysteroid titer and morph-specific reproduction in the wing-polymorphic cricket, Gryllus firmus. Evolution 55, 538-549.

Zera, A.J., Cisper, G., 2001. Genetic and diurnal variation in the juvenile hormone titer in a wing-polymorphic cricket: Implications for the evolution of life histories and dispersal. Physiological and Biochemical Zoology 74, 293-306.

Zera, A.J., Denno, R.F., 1997. Physiology and ecology of dispersal polymorphism in insects. Annual Review of Entomology 42, 207-230.

Zera, A.J., Harshman, L.G., 2001. Physiology of life history trade-offs in animals. Annual Review of Ecology and Systematics 32, 95-126.

Zera, A.J., Larsen, A., 2001. The metabolic basis of life history variation: Genetic and phenotypic differences in lipid reserves among life history morphs of the wing-polymorphic cricket, Gryllus firmus. Journal of Insect Physiology 47, 1147-1160.

Zera, A.J., Zhao, Z., 2003a. Life history evolution and the microevolution of intermediary metabolism: Activities of lipid-metabolizing enzymes in life-history morphs of a wing-dimorphic cricket. Evolution 57, 568-596.

Zera, A.J., Zhao, Z., 2003b. Morph-dependent fatty-acid oxidation in a wingpolymorphic cricket: Implications for morph specialization for dispersal vs. reproduction. Journal of Insect Physiology 49, 933-943.

Zera, A.J., Zhao, Z., 2004. Effect of a juvenile hormone analogue on lipid metabolism in a wing-polymorphic cricket: Implications for the biochemical basis of the trade-off between reproduction and dispersal. Biochemical and Physiological Zoology 77, 255-266.

Zera, A.J., Sall, J., Grudzinski, K., 1997. Flight-muscle polymorphism in the cricket Gryllus firmus: Muscle characteristics and their influence on the evolution of flightlessness. Physiological Zoology 70, 519-529.

Zera, A.J., Potts, J., Kobus, K., 1998. The physiology of life history tradeoffs: Experimental analysis of a hormonally induced life history trade-off in Gryllus assimilis. American Naturalist 152, 7-23.

Zera, A.J., Sall, J., Otto, K., 1999. Biochemical aspects of flight and flightlessness in Gryllus: flight fuels, enzyme activities, and electrophoretic profiles of flight muscles from flight-capable and flightless morphs. Journal of Insect Physiology 45, 275-285.

Zhao, Z., Zera, A.J., 2001. Enzymological and radiotracer studies of lipid metabolism in the flight-capable and flightless morphs of the wingpolymorphic cricket, Gryllus firmus. Journal of Insect Physiology 47, 1337-1347.

Zhao, Z., Zera, A.J., 2002. Differential lipid biosynthesis underlies a trade-off between reproduction and flight capability in a wing-polymorphic cricket. Proceedings of the National Academy of Sciences USA 99, 1682916834 